\title{
Cingulum bundle integrity associated with delusions of control in schizophrenia: Preliminary evidence from diffusion-tensor tractography
}

\section{Citation}

Whitford, Thomas J., Marek Kubicki, Paula E. Pelavin, Diandra Lucia, Jason S. Schneiderman, Christos Pantelis, Robert W. McCarley, and Martha E. Shenton. 2015. “Cingulum Bundle Integrity Associated with Delusions of Control in Schizophrenia: Preliminary Evidence from Diffusion-Tensor Tractography." Schizophrenia Research 161 (1) (January): 36-41. doi:10.1016/ j.schres.2014.08.033.

\section{Published Version}

doi:10.1016/j.schres.2014.08.033

\section{Permanent link}

http://nrs.harvard.edu/urn-3:HUL.InstRepos:28538483

\section{Terms of Use}

This article was downloaded from Harvard University's DASH repository, and is made available under the terms and conditions applicable to Open Access Policy Articles, as set forth at http:// nrs.harvard.edu/urn-3:HUL.InstRepos:dash.current.terms-of-use\#OAP

\section{Share Your Story}

The Harvard community has made this article openly available. Please share how this access benefits you. Submit a story. 


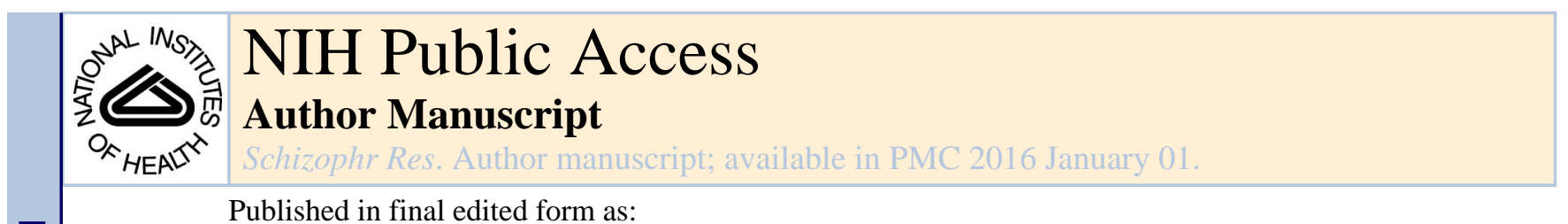

Published in final edited form as:

Schizophr Res. 2015 January ; 161(1): 36-41. doi:10.1016/j.schres.2014.08.033.

\title{
Cingulum Bundle Integrity Associated with Delusions of Control in Schizophrenia: Preliminary Evidence from Diffusion-Tensor Tractography
}

\author{
Thomas J. Whitford, Ph.D. ${ }^{1,2,3}$, Marek Kubicki, M.D., Ph.D. ${ }^{2}$, Paula E. Pelavin, B.Sc. ${ }^{2}$, \\ Diandra Lucia, B.Sc. ${ }^{2}$, Jason S. Schneiderman, Ph.D. ${ }^{2}$, Christos Pantelis, M.D., Ph.D. ${ }^{3}$, \\ Robert W. McCarley, M.D., Ph.D ${ }^{4}$, and Martha E. Shenton, Ph.D. 2,4 \\ ${ }^{1}$ School of Psychology, University of New South Wales, Sydney, NSW, Australia \\ 2Psychiatry Neuroimaging Laboratory, Department of Psychiatry, Brigham and Women's Hospital, \\ Harvard Medical School, Boston, MA, USA \\ ${ }^{3}$ Melbourne Neuropsychiatry Centre, Department of Psychiatry, University of Melbourne, \\ Melbourne, VIC, Australia \\ ${ }^{4}$ Clinical Neuroscience Division, Laboratory of Neuroscience, Department of Psychiatry, Veterans \\ Affairs Boston Healthcare System, Harvard Medical School, Brockton, MA, USA
}

\begin{abstract}
Background-Delusions of control are among the most distinctive and characteristic symptoms of schizophrenia. Several theories have been proposed that implicate aberrant communication between spatially disparate brain regions in the etiology of this symptom. Given that white matter fasciculi represent the anatomical infrastructure for long-distance communication in the brain, the present study investigated whether delusions of control were associated with structural abnormalities in four major white matter fasciculi.
\end{abstract}

Methods-Ten schizophrenia patients with current delusions of control, 13 patients with no clinical history of delusions of control, and 12 healthy controls underwent a Diffusion-Tensor Imaging (DTI) scan. Deterministic tractography was used to extract the corpus callosum, superior longitudinal fasciculus, arcuate fasciculus and cingulum bundle. The structural integrity of these four fasciculi were quantified with Fractional Anisotropy (FA), and compared between groups.

\footnotetext{
(C) 2014 Elsevier B.V. All rights reserved.

*Corresponding author: Thomas J. Whitford, School of Psychology, Mathews Building, University of New South Wales (UNSW), Sydney, NSW, 2052, Australia, Phone: +61293853936, t.whitford@unsw.edu.au.

Contributors

TJW designed the study, analyzed the data and wrote the first draft of the manuscript. MK and MES designed the study, collected and analyzed the data and helped interpret the findings. PEP and DL assisted with image pre-processing and analysis. JSS, CP and RWM assisted with analyzing the data and interpreting the findings. All authors contributed to and have approved the final manuscript.

Conflict of Interest

All authors declare that they have no conflicts of interest.

Publisher's Disclaimer: This is a PDF file of an unedited manuscript that has been accepted for publication. As a service to our customers we are providing this early version of the manuscript. The manuscript will undergo copyediting, typesetting, and review of the resulting proof before it is published in its final citable form. Please note that during the production process errors may be discovered which could affect the content, and all legal disclaimers that apply to the journal pertain.
} 
Results-The patients with delusions of control exhibited significantly lower FA in all four fasciculi, relative to the healthy controls. Furthermore, the patients with delusions of control also exhibited significantly lower FA in the cingulum bundle relative to patients without a history of this symptom, and this difference remained significant when controlling for between-group differences in global SAPS score and medication dosage.

Conclusions-The results suggest that structural damage to the cingulum bundle may be involved in the etiology of delusions of control, possibly because of its role in connecting the action initiation areas of the premotor cortex with the cingulate gyrus.

\section{Keywords}

schizophrenia; delusions of control; passivity experiences; Diffusion-Tensor Imaging (DTI); tractography; cinglulum bundle

\section{INTRODUCTION}

Delusions of control are among the most distinctive and characteristic symptoms of schizophrenia. Delusions of control refer to the belief, or arguably the experience (Fletcher and Frith, 2009), that one's own actions are being generated or manipulated by an external agent. Spence et al. (Spence et al., 1997) quoted a patient with schizophrenia describing her experience of delusions of control during the performance of a willed hand movement: $-I$ felt like an automaton, guided by a female spirit who had entered me during it. No voice-a feeling.

While the underlying causes of delusions of control remain a matter of debate, several theories have been proposed as to their etiology. Hoffman and McGlashan (Hoffman and McGlashan, 1993) have suggested that delusions of control are caused by a "breakdown in communication between cortical areas" (p.119) causing certain neural modules (or "parasitic foci") to output independently of input, thus causing the generation of unintended actions. Frith, Blakemore and Wolpert (Frith et al., 2000), in contrast, have argued that delusions of control are caused by abnormal inhibitory control of the parietal cortex by the frontal lobe. According to this theory, this abnormality in frontal-parietal communication leads to selfgenerated actions being misperceived as externally-generated. In a somewhat similar vein, Crow (Crow, 1998) has suggested that delusions of control arise from abnormal interhemispheric interactions between lateralized, language-related neural modules that are involved in differentiating the self from the external world.

Although these theories differ from each other in several respects, a notable common feature is that they all implicate abnormal communication between spatially disparate brain regions in the etiology of delusions of control. Given that the white matter (WM) fasciculi represent the anatomical infrastructure for long-distance communication in the brain (i.e., communication between neural modules separated by more than a couple of centimeters), the question arises as to whether delusions of control could ultimately be underpinned by structural abnormalities to the connecting WM fasciculi (Whitford et al., 2012), as opposed to structural abnormalities to the gray matter regions connected by these fasciculi per se. However, while a number of studies have identified WM abnormalities in patients with 
schizophrenia, including with electron microscopy (Uranova et al., 2007), structural MRI (Hulshoff Pol et al., 2004a; Hulshoff Pol et al., 2004b; Whitford et al., 2007) and DiffusionTensor Imaging (DTI) (Karlsgodt et al., 2008; Price et al., 2007; Skelly et al., 2008; Zalesky et al., 2011), there have been no studies (to our knowledge) that have specifically investigated the WM abnormalities associated with delusions of control. Categorizing patients on the basis of a specific clinical symptom is potentially more sensitive than the traditional approach of comparing healthy controls with a clinically heterogeneous sample of schizophrenia patients who can potentially not have a single symptom in common. This approach of categorizing patients based on their symptom profile has, for example, previously been used to identify WM abnormalities in the corpus callosum (Hubl et al., 2004; Mulert et al., 2012) and arcuate fasciculus (Catani et al., 2011) in schizophrenia patients who had or were prone to auditory hallucinations.

The aim of the present study was to investigate the WM underpinnings of delusions of control in patients with schizophrenia. Specifically, the present study used DTI in combination with deterministic tractography to test the hypothesis that delusions of control are associated with structural damage to the WM fasciculi. We hypothesized that: (1) schizophrenia patients who were currently experiencing delusions of control would exhibit structural abnormalities (i.e., relative to healthy controls) in one or more of four major WM fasciculi, namely the corpus callosum, superior longitudinal fasciculus, arcuate fasciculus and cingulum bundle, and that (2) these WM abnormalities would be more pronounced in patients currently experiencing delusions of control, compared to schizophrenia patients who had never experienced this specific clinical symptom.

\section{METHOD}

\subsection{Participants}

Twenty-three patients with chronic schizophrenia were recruited from inpatient, outpatient, day-treatment and foster care programs at the Veterans Administration Boston Healthcare System, Brockton, Massachusetts. The diagnosis of schizophrenia was made according to DSM-IV criteria, based on the Structured Clinical Interview for DSM-IV (SCID; (First et al., 1997)) and a review of the medical record. Ten of the 23 schizophrenia participants were currently presenting with delusions of control at the time of scanning - these participants constituted the Delusions-of-Control (DOC) group. The presence of delusions of control was quantified as a score $\geq 2$ on the 'delusions of being controlled' item of the Scale for the Assessment of Positive Symptoms(SAPS; (Andreasen, 1984)). The remaining 13 schizophrenia participants were not presenting with delusions of control at the time of scanning (i.e., scored zero on the 'delusions of being controlled' item of the SAPS), and reported never having experienced delusions of control at any time in their life - these participants constituted the No-Delusions-of-Control (nDOC) group. The DOC and nDOC groups were compared to a group of 12 healthy control (HC) participants, who were recruited from the general community. The demographic details for the DOC, nDOC and HC participants are summarized in Table 1. The three groups were matched for age, gender, handedness (Oldfield, 1971) and estimated premorbid IQ (Wilkinson, 1993), although they differed on parental socio-economic status (Hollingshead, 1965). 
Exclusion criteria for all participants were: left handedness, a history of neurological illness including epilepsy, a history of steroid use or electroconvulsive shock therapy, a lifetime history of substance dependence or a history of substance abuse within the past 5 years, and estimated premorbid IQ $<75$. Furthermore, HC participants were also screened for the presence of an Axis I disorder with the SCID Non-Patient Edition. HC participants were also excluded if they reported having a first-degree relative with an Axis I disorder. The study was approved by the Harvard Medical School Internal Review Board, the Brigham and Women's Hospital Human Subjects Committee, and the Veterans Affairs Boston Healthcare System. After a detailed description of the study, all participants gave written informed consent to participate.

\subsection{Diffusion-Tensor Imaging (DTI) Acquisition}

Diffusion-weighted images were acquired on a 1.5 Tesla GE scanner with a quadrature head coil. A line scan diffusion imaging protocol (LSDI) was employed (Kubicki et al., 2002), with the following parameters: 6 orthogonal gradient directions with high diffusion weighting ( $b=1000 \mathrm{sec} / \mathrm{mm} 2), 2$ images with low diffusion weighting ( $b=5 \mathrm{sec} / \mathrm{mm} 2)$, FOV $=220 \times 165 \mathrm{~mm}$, matrix $=128 \times 128 \mathrm{~mm}$, in-plane resolution $=1.7 \times 1.7 \mathrm{~mm}$, slice thickness $=4 \mathrm{~mm}$, interslice distance $=1 \mathrm{~mm}$, echo time $=64 \mathrm{msec}$, effective repetition time $=2592 \mathrm{msec}, \mathrm{NEX}=1$, receiver bandwidth $=4 \mathrm{kHz}, 31-35$ coronal slices to provide full brain coverage, acquisition time $=$ approximately 30 minutes. Diffusion-Tensor Images (DTIs) were constructed from the diffusion-weighted images using a least-squares estimation method. Fractional Anisotropy (FA), a standard diffusion metric of white matter integrity (Basser and Pierpaoli, 1996), was calculated at every voxel in the constructed DTIs.

\subsection{Diffusion-Tensor Imaging (DTI) Analysis}

All image analysis was performed with the 3D-Slicer software package (http:// www.slicer.org, Surgical Planning Laboratory, Brigham and Women's Hospital, Boston, Massachusetts). Four white matter fasciculi, namely the corpus callosum, superior longitudinal fasciculus (SLF), arcuate fasciculus and cingulum bundle, were extracted from the DTIs with deterministic (streamline) tractography. Regions of interest (ROIs), based on the atlas of Mori et al. (Mori et al., 2005), were manually drawn on each participants FA image, with color-by-orientation images being used to assist with ROI placement. The ROIs were all drawn by a single rater (PEP or DL) and confirmed by a second rater (MK). The ROIs and extracted fibers for the 4 fasciculi are depicted in Figure 1.

The protocols for drawing the 4 ROIs were as follows: the corpus callosum was composed of a single ROI traced on the red voxels of the midsagittal slice of the color-by-orientation image. The superior longitudinal fasciculus was defined using a posterior inclusion ROI, which was placed on the green voxels at the level of the fornix, and an anterior inclusion ROI that was defined by following the fibers anteriorly until they projected laterally towards the cortex (red/orange voxels). An axial exclusion ROI was also placed at the level of the arcuate where it projected inferiorly to the temporal lobe (blue voxels). The arcuate fasciculus was defined on 5-10 axial slices, beginning two slices superior to the dorsal-most slice of the corpus callosum and moving inferiorly, and was drawn on the green voxels 
lateral to the corona radiata. The cingulum bundle was defined using four coronal inclusion ROIs and a midsagittal exclusion ROI to remove commissural fibers. The anterior inclusion ROI was placed on three consecutive slices $3-5$ slices posterior to the genu, while the posterior inclusion ROI was drawn on a single coronal slice 2-4 slices posterior to the splenium. The two intermediate inclusion ROIs were placed on the same coronal slice, immediately superior and inferior to the splenium.

The voxels defined by the ROIs were subsequently used as seedpoints for tractography. The defined voxels were each seeded 5 times, with each seeding initiated from random point within the voxel. Tractography was based on a Runge-Kutta second-order protocol and followed the direction defined by the principle eigenvector. The following parameters were used: step size $=0.5 \mathrm{~mm}$, stopping threshold $=\mathrm{FA}<0.15$, maximum radius of curvature $=$ $0.87 \mathrm{~mm}$. FA was sampled at every step on the extracted streamlines for each of the WM fasciculi. Mean FA was calculated for fasciculus, for each participant, and used in the statistical analysis. Inter-rater reliability was calculated as the intra-class correlation of each tract's mean FA calculated from 5 randomly selected participants. The ICCs were 0.959 for the corpus callosum, 0.954 for the arcuate fasciculus, 0.917 for the superior longitudinal fasciculus and 0.840 for the cingulum bundle.

\subsection{Statistical analysis}

All analyses were performed in SPSS version 17 (www.spss.com). Univariate ANOVAs were used to investigate the effects of Diagnosis (3 groups: DOC, nDOC and HC) on FA for each of the four fasciculi. Alpha was set at 0.0125 (i.e., 0.05/4), after Bonferroni correction for multiple comparisons. Tukey's Honestly Significant Difference (HSD) post-hoc contrasts were used to identify the simple effects underlying any significant main effects.

\section{RESULTS}

Figure 2 shows the level of Fractional Anisotropy (FA) in the corpus callosum (Fig. 2A), superior longitudinal fasciculus (Fig. 2B), arcuate fasciculus (Fig. 2C) and cingulum bundle (Fig. 2D) in schizophrenia patients with current delusions of control (DOC), schizophrenia patients without a history of delusions of control (nDOC) and healthy control participants (HC). After Bonferroni correction for multiple comparisons, the ANOVAs revealed significant differences between the three clinical groups in the FA of all four WM fasciculi. Specifically:

There was a significant between-group difference in the FA of the corpus callosum $\left(\mathrm{F}_{2,32}=8.289, \mathrm{p}=.001\right.$ eta $\left.^{2}=.364\right)$. Post-hoc contrasts (Tukey's HSD) revealed significant FA differences between the DOC and HC groups ( $\mathrm{p}=.002$, Cohen's $\mathrm{d}=1.65)$, and between the nDOC and HC groups ( $\mathrm{p}=.020$, Cohen's $\mathrm{d}=1.10$ ), but not between the DOC and $\mathrm{nDOC}$ groups $(\mathrm{p}=.463$, Cohen's $\mathrm{d}=0.71)$.

There was a significant between-group difference in the FA of the superior longitudinal fasciculus $\left(\mathrm{F}_{2,32}=6.335, \mathrm{p}=.005\right.$, eta $\left.{ }^{2}=.304\right)$. Post-hoc contrasts revealed significant $\mathrm{FA}$ differences between the DOC and HC groups ( $\mathrm{p}=.009$, Cohen's $\mathrm{d}=1.43$ ), and between the 
$\mathrm{nDOC}$ and $\mathrm{HC}$ groups (mean difference $\mathrm{p}=.018$, Cohen's $\mathrm{d}=1.11$ ), but not between the DOC and nDOC groups ( $\mathrm{p}=.878$, Cohen's $\mathrm{d}=0.25)$.

There was a significant between-group difference in the FA of the arcuate fasciculus $\left(\mathrm{F}_{2,32}=8.072, \mathrm{p}=.002\right.$ eta $\left.^{2}=.358\right)$. Post-hoc contrasts revealed a significant FA difference between the DOC and HC groups ( $\mathrm{p}=.001$, Cohen's $\mathrm{d}=1.64$ ), but not between the $\mathrm{nDOC}$ and HC groups ( $\mathrm{p}=.075$, Cohen's $\mathrm{d}=1.04)$ or between the DOC and nDOC groups $(\mathrm{p}=.151$, Cohen's d $=0.81$ ).

There was a significant between-group difference in the FA of the cingulum bundle $\left(\mathrm{F}_{2,32}=7.518, \mathrm{p}=.002\right.$ eta $\left.^{2}=.341\right)$. Post-hoc contrasts revealed a significant difference in FA between the DOC and HC groups ( $\mathrm{p}=.003$, Cohen's $\mathrm{d}=1.39$ ), but not between the $\mathrm{nDOC}$ and HC groups $(\mathrm{p}=.816$, Cohen's $\mathrm{d}=0.32)$. A significant difference was also observed between the DOC and nDOC groups ( $\mathrm{p}=.011$, Cohen's $\mathrm{d}=1.25$ ), with the DOC participants exhibiting significantly reduced levels of cingulum FA relative to the $\mathrm{nDOC}$ participants (see Fig 2). As shown in Table 1, the DOC participants had significantly higher SAPS scores, and non-significantly higher medication dosages than the nDOC participants. This raised the possibility that the differences in cingulum FA were being driven by betweengroup differences in medication exposure or psychotic symptom severity generally, as opposed to delusions of control specifically. However, this possibility was ameliorated by the fact that the difference between the DOC and nDOC groups on cingulum FA remained significant when controlling between-group differences in SAPS score and CPZ-equivalent medication dosage $\left(\mathrm{F}_{1,20}=11.399, \mathrm{p}=.004\right.$ eta $\left.^{2}=.416\right)$. A significant negative correlation was, however, observed between SZ participants' CPZ-equivalent medication dosage and their FA in the cingulum bundle $(\mathrm{r}(21)=-.467, \mathrm{p}=.033)$. Non-significant correlations were also observed between SZ patients' CPZ-equivalent medication dosage and their FA in the corpus callosum $(\mathrm{r}(21)=-.172, \mathrm{p}=.457)$, superior longitudinal fasciculus $(\mathrm{r}(21)=-.342, \mathrm{p}=$. $129)$ and arcuate fasciculus $(\mathrm{r}(21)=-.348, \mathrm{p}=.128)$.

\section{DISCUSSION}

The results of the study revealed that schizophrenia patients who were currently presenting with delusions of control (the DOC group) exhibited significantly lower FA in the corpus callosum, superior longitudinal fasciculus, arcuate fasciculus and cingulum bundle relative to matched healthy controls (HC). The schizophrenia patients without a history of delusions of control (the nDOC group) exhibited FA levels that were intermediate between the DOC and HC participants for all WM four fasciculi. Finally, the DOC participants exhibited significantly lower levels of $\mathrm{FA}$ in the cingulum bundle relative to $\mathrm{nDOC}$ participants, after controlling for between-group differences in SAPS score and CPZ-equivalent medication dosage. This result suggests that structural abnormalities in the cingulum bundle could potentially contribute to the development of delusions of control.

Several previous studies have used DTI to identify structural abnormalities in the white matter in patients with schizophrenia, including in the corpus callosum (Whitford et al., 2010), arcuate fasciculus (Phillips et al., 2009), superior longitudinal fasciculus (Karlsgodt et al., 2008; Shergill et al., 2007) and cingulum bundle (Kubicki et al., 2003). Furthermore, 
some WM abnormalities have been found to specific to schizophrenia patients with certain clinical symptoms relative to patients without these symptoms, including FA reductions in the temporoparietal fibers of the corpus callosum in patients prone to auditory hallucinations (Hubl et al., 2004; Mulert et al., 2012), and FA increases in the white matter of the frontal cortex, cingulate gyrus and basal ganglia in patients with passivity experiences (Sim et al., 2009). Gray matter abnormalities, particularly in the parietal and frontal cortices, have also been identified in patients with passivity experiences and other first-rank Symptoms, relative to patients without these symptoms (Maruff et al., 2005). However, to the best of our knowledge, the present study is the first to explicitly identify white matter abnormalities that are specific to schizophrenia patients with a clinical history of delusions of control.

The fact that our patients with delusions of control exhibited significantly lower FA in the cingulum bundle, but not the corpus callosum, superior longitudinal fasciculus or arcuate fasciculus, compared to schizophrenia patients without a history of delusions of control, suggests that the cingulum bundle may play a role in the etiology of these particular delusions. This result is also consistent with the results of a recent study by Whitford et al. (2014) who identified a significant negative correlation between patients' score on the global Delusions scale of the SAPS and their FA in a sub-component of the cingulum bundle. The cingulum bundle connects the cingulate gyrus with several cerebral regions, including the premotor, prefrontal and parietal cortices, thalamus and hippocampus (Pandya et al., 1981). Consequently, damage to the cingulum bundle has been associated with a number of cognitive abnormalities including with attention, memory and emotional processing (Vogt and Laureys, 2005). The efferent connections between the anterior cingulate gyrus and the premotor cortex may be of particular relevance to delusions of control, in keeping with the 'self-monitoring' models of schizophrenia (Feinberg, 1978). Specifically, it is possible that abnormal communication between the premotor cortex which is thought to be involved in the initiation of willed actions (Picard and Strick, 2001) and the anterior cingulate cortex - which is thought to be involved in error monitoring (Carter et al., 1998) - could lead to prediction errors during the performance of willed actions. These prediction errors, and the corresponding activation of the dopamine system, could potentially lead to willed actions feeling unpredictable and abnormally salient, which could lead to them being misattributed to an external force (Corlett et al., 2010; Frith et al., 2000; Kapur, 2003; Roiser et al., 2009). It should, however, be emphasized that the significant difference between the DOC and nDOC groups in terms of their level of cingulum FA was due less to the DOC group exhibiting severely abnormal levels of cingulum FA (i.e., relative to their level of FA abnormality in the other fasciculi) but rather to the $n D O C$ group exhibiting relatively normal levels of FA in the cingulum - see Figure 2D. Indeed, it is notable that there were no significant differences between the nDOC and healthy control groups in terms of cingulum FA; that is, cingulum abnormalities were specific to the participants suffering from delusions of control. This result opens up the intriguing possibility that abnormalities in the cingulum may be necessary for the development of delusions of control, and also highlights the sensitivity of our approach of dividing patients (all with a common diagnosis of schizophrenia) into sub-groups on the basis of their symptom profile. Investigating whether structural abnormalities in the cingulum are necessary and/or sufficient for the development of delusions of control (both in 
patients with schizophrenia and in people with other psychotic disorders) would, we suggest, be a fruitful area for future research.

There were at least three limitations to the present study. The first relates to the small sample size, which was due primarily to the difficulties inherent in recruiting chronically ill schizophrenia patients who were currently presenting with clear and frank delusions of control. Despite the fact that the observed effect sizes were large (i.e., eta ${ }^{2}>.3$ for all analyses, and Cohen's $\mathrm{d}>1$ for all significant contrasts), and the results significant after appropriate correction for multiple comparisons, it is possible that the recruited participants were not representative of their respective clinical populations. It would thus be advantageous if the results were replicated in a larger sample of participants. The second limitation relates to the fact that all of the schizophrenia participants were either currently being treated or had previously been treated with antipsychotic medication. This is problematic in light of evidence indicating that exposure to antipsychotic medication may influence brain structure, including WM, in and of itself (Konopaske et al., 2008), and the fact that, in the present study, a significant correlation was observed between patients' CPZequivalent medication dosage and their level of FA in the cingulum bundle. Despite the fact that in the present study: (1) there was no significant difference between the DOC and nDOC groups in terms of CPZ-equivalent medication dosage, and (2) the between-group difference in cingulum FA remained statistically significant when controlling for CPZequivalent medication dosage, it would nevertheless be useful if the results were replicated in a sample of neuroleptic-naïve participants. Furthermore, notwithstanding our attempts to statistically control for between-group differences in medication and positive symptom severity, it is important to acknowledge that the more severe FA abnormalities exhibited by DOC group could potentially have been caused (at least in part) by auxiliary clinical factors such as (for example) the DOC patients being less responsive to antipsychotic medication. Finally, it must also be acknowledged that the DTI sequence used in the present study is now considered to be of relatively low-resolution. While our validated manual-tractography procedure ensured that the four fasciculi-of-interest were accurately identified in every participant, the low-resolution sequence meant that the extracted fasciculi were sparsely fibered and somewhat 'blocky' in appearance - as can be seen most clearly in the example corpus callosi shown in Figure 1. It would thus be advantageous if the results of the present study were replicated in a larger sample and with a more recent and high-resolution DTI sequence, such as HARDI (High Angular Resolution Diffusion Weighted Imaging).

In summary, the present study identified structural WM abnormalities, as quantified with the Diffusion-Tensor Imaging metric of Fractional Anisotropy, in the corpus callosum, superior longitudinal fasciculus, arcuate fasciculus and cingulum bundle in patients with chronic schizophrenia who were currently presenting with delusions of control. Furthermore, patients with delusions of control also exhibited significantly lower FA levels in the cingulum bundle relative to a matched group of schizophrenia patients who had no history of delusions of control. In addition to supplementing the growing body of evidence indicating the presence of structural WM abnormalities in patients with schizophrenia, these results suggest that structural damage to the cingulum bundle could potentially be involved in the development of delusions of control. 


\section{Acknowledgments}

Funding Body Agreement and Policies

This work was supported by the National Health and Medical Research Council of Australia (520627 to TJW; 628386 to CP), the NARSAD Brain and Behavior Research Foundation (17537 to TJW), the National Institutes of Health (R03 MH068464-0; U54 EB005149; R01 MH 50747 and K05 MH 070047; T32 MH 016259; P50 MH 080272), the Department of Veterans Affairs (VA Merit Award, VA Research Enhancement Award Program, and VA Schizophrenia Research Center Grant), and the National Institute for Mental Health (MH 040799).

The authors would like to thank Mr Tali Swisher for his assistance with image pre-processing.

\section{References}

Andreasen, NC. Scale for the Assessment of Positive Symptoms (SAPS). Iowa U; Iowa City: 1984.

Basser PJ, Pierpaoli C. Microstructural and physiological features of tissues elucidated by quantitativediffusion-tensor MRI. J Magn Reson B. 1996; 111(3):209-219. [PubMed: 8661285]

Carter CS, Braver TS, Barch DM, Botvinick MM, Noll D, Cohen JD. Anterior cingulate cortex, error detection, and the online monitoring of performance. Science. 1998; 280(5364):747-749. [PubMed: 9563953]

Catani M, Craig MC, Forkel SJ, Kanaan R, Picchioni M, Toulopoulou T, Shergill S, Williams S, Murphy DG, McGuire P. Altered Integrity of Perisylvian Language Pathways in Schizophrenia: Relationship to Auditory Hallucinations. Biol Psychiatry. 2011; 70(12):1143-1150. [PubMed: 21798516]

Corlett PR, Taylor JR, Wang XJ, Fletcher PC, Krystal JH. Toward a neurobiology of delusions. Prog Neurobiol. 2010; 92(3):345-369. [PubMed: 20558235]

Crow T. Schizophrenia as a transcallosal misconnection syndrome. Schizophr Res. 1998; 30(2):111114. [PubMed: 9549773]

Feinberg I. Efference copy and corollary discharge: implications for thinking and its disorders. Schizophrenia Bulletin. 1978; 4(4):636-640. [PubMed: 734369]

First, M.; Spitzer, R.; Gibbon, M.; Williams, J. Structured Clinical Interview for DSM-IV-TR Axis I Disorders (SCID-I), Clinician Version. American Psychiatric Publishing; 1997.

Fletcher P, Frith C. Perceiving is believing: a Bayesian approach to explaining the positive symptoms of schizophrenia. Nat Rev Neurosci. 2009; 10(1):48-58. [PubMed: 19050712]

Frith CD, Blakemore S, Wolpert DM. Explaining the symptoms of schizophrenia: abnormalities in the awareness of action. Brain Research Reviews. 2000; 31:357-363. [PubMed: 10719163]

Hoffman RE, McGlashan TH. Parallel distributed processing and the emergence of schizophrenic symptoms. Schizophr Bull. 1993; 19(1):119-140. [PubMed: 8451607]

Hollingshead, A. Two factor of index of social position. Yale Station; New Haven: 1965.

Hubl D, Koenig T, Strik W, Federspiel A, Kreis R, Boesch C, Maier SE, Schroth G, Lovblad K, Dierks T. Pathways that make voices: white matter changes in auditory hallucinations. Arch Gen Psychiatry. 2004; 61(7):658-668. [PubMed: 15237078]

Hulshoff Pol HE, Brans RG, van Haren NE, Schnack HG, Langen M, Baare WF, van Oel CJ, Kahn RS. Gray and white matter volume abnormalities in monozygotic and same-gender dizygotic twins discordant for schizophrenia. Biol Psychiatry. 2004a; 55(2):126-130. [PubMed: 14732591]

Hulshoff Pol HE, Schnack HG, Mandl RC, Cahn W, Collins DL, Evans AC, Kahn RS. Focal white matter density changes in schizophrenia: reduced inter-hemispheric connectivity. Neuroimage. 2004b; 21(1):27-35. [PubMed: 14741639]

Kapur S. Psychosis as a state of aberrant salience: a framework linking biology, phenomenology, and pharmacology in schizophrenia. Am J Psychiatry. 2003; 160(1):13-23. [PubMed: 12505794]

Karlsgodt K, van Erp T, Poldrack R, Bearden C, Nuechterlein K, Cannon T. Diffusion tensor imaging of the superior longitudinal fasciculus and working memory in recent-onset schizophrenia. Biol Psychiatry. 2008; 63(5):512-518. [PubMed: 17720147] 
Konopaske G, Dorph-Petersen K, Sweet R, Pierri J, Zhang W, Sampson A, Lewis D. Effect of chronic antipsychotic exposure on astrocyte and oligodendrocyte numbers in macaque monkeys. Biol Psychiatry. 2008; 63(8):759-765. [PubMed: 17945195]

Kubicki M, Westin CF, Maier SE, Frumin M, Nestor PG, Salisbury DF, Kikinis R, Jolesz FA, McCarley RW, Shenton ME. Uncinate fasciculus findings in schizophrenia: a magnetic resonance diffusion tensor imaging study. Am J Psychiatry. 2002; 159(5):813-820. [PubMed: 11986136]

Kubicki M, Westin CF, Nestor PG, Wible CG, Frumin M, Maier SE, Kikinis R, Jolesz FA, McCarley $\mathrm{RW}$, Shenton ME. Cingulate fasciculus integrity disruption in schizophrenia: a magnetic resonance diffusion tensor imaging study. Biol Psychiatry. 2003; 54(11):1171-1180. [PubMed: 14643084]

Maruff P, Wood SJ, Velakoulis D, Smith DJ, Soulsby B, Suckling J, Bullmore ET, Pantelis C. Reduced volume of parietal and frontal association areas in patients with schizophrenia characterized by passivity delusions. Psychol Med. 2005; 35(6):783-789. [PubMed: 15997599]

Mori, S.; Wakana, S.; van Zijl, PC.; Nagae-Poetscher, LM. MRI Atlas of Human White Matter. Elsevier; 2005.

Mulert C, Kirsch V, Whitford TJ, Alvarado J, Pelavin P, McCarley RW, Kubicki M, Salisbury DF, Shenton ME. Hearing voices: a role of interhemispheric auditory connectivity? World J Biol Psychiatry. 2012; 13(2):153-158. [PubMed: 21623667]

Oldfield RC. The assessment and analysis of handedness: the Edinburgh inventory. Neuropsychologia. 1971; 9(1):97-113. [PubMed: 5146491]

Pandya DN, Van Hoesen GW, Mesulam MM. Efferent connections of the cingulate gyrus in the rhesus monkey. Exp Brain Res. 1981; 42(3-4):319-330. [PubMed: 6165607]

Phillips O, Nuechterlein K, Clark K, Hamilton L, Asarnow R, Hageman N, Toga A, Narr K. Fiber tractography reveals disruption of temporal lobe white matter tracts in schizophrenia. Schizophr Res. 2009; 107(1):30-38. [PubMed: 19028423]

Picard N, Strick P. Imaging the premotor areas. Curr Opin Neurobiol. 2001; 11(6):663-672. [PubMed: 11741015]

Price G, Cercignani M, Parker G, Altmann D, Barnes T, Barker G, Joyce E, Ron M. Abnormal brain connectivity in first-episode psychosis: a diffusion MRI tractography study of the corpus callosum. Neuroimage. 2007; 35(2):458-466. [PubMed: 17275337]

Roiser J, Stephan K, den Ouden H, Barnes T, Friston K, Joyce E. Do patients with schizophrenia exhibit aberrant salience? Psychol Med. 2009; 39(2):199-209. [PubMed: 18588739]

Shergill S, Kanaan R, Chitnis X, O'Daly O, Jones D, Frangou S, Williams S, Howard R, Barker G, Murray R, McGuire P. A diffusion tensor imaging study of fasciculi in schizophrenia. Am J Psychiatry. 2007; 164(3):467-473. [PubMed: 17329472]

Sim K, Yang GL, Loh D, Poon LY, Sitoh YY, Verma S, Keefe R, Collinson S, Chong SA, Heckers S, Nowinski W, Pantelis C. White matter abnormalities and neurocognitive deficits associated with the passivity phenomenon in schizophrenia: a diffusion tensor imaging study. Psychiatry Res. 2009; 172(2):121-127. [PubMed: 19297135]

Skelly L, Calhoun V, Meda S, Kim J, Mathalon D, Pearlson G. Diffusion tensor imaging in schizophrenia: relationship to symptoms. Schizophr Res. 2008; 98(1-3):157-162. [PubMed: 18031994]

Spence SA, Brooks DJ, Hirsch SR, Liddle PF, Meehan J, Grasby PM. A PET study of voluntary movement in schizophrenic patients experiencing passivity phenomena (delusions of alien control). Brain. 1997; 120:1997-2011. [PubMed: 9397017]

Uranova N, Vostrikov V, Vikhreva O, Zimina I, Kolomeets N, Orlovskaya D. The role of oligodendrocyte pathology in schizophrenia. Int J Neuropsychopharmacol. 2007; 10(4):537-545. [PubMed: 17313698]

Vogt BA, Laureys S. Posterior cingulate, precuneal and retrosplenial cortices: cytology and components of the neural network correlates of consciousness. Prog Brain Res. 2005; 150:205217. [PubMed: 16186025]

Whitford TJ, Ford JM, Mathalon DH, Kubicki M, Shenton ME. Schizophrenia, myelination, and delayed corollary discharges: a hypothesis. Schizophr Bull. 2012; 38(3):486-494. [PubMed: 20855415] 
Whitford TJ, Grieve SM, Farrow TF, Gomes L, Brennan J, Harris AWF, Gordon E, Williams LM. Volumetric white matter abnormalities in first-episode schizophrenia: a longitudinal, tensor-based morphometry study. American Journal of Psychiatry. 2007; 164:1082-1089. [PubMed: 17606660]

Whitford TJ, Kubicki M, Schneiderman JS, O’Donnell LJ, King R, Alvarado JL, Khan U, Markant D, Nestor PG, Niznikiewicz M, McCarley RW, Westin CF, Shenton ME. Corpus callosum abnormalities and their association with psychotic symptoms in patients with schizophrenia. Biological Psychiatry. 2010; 68:70-77. [PubMed: 20494336]

Whitford TJ, Lee SW, Oh JS, de Luis-Garcia R, Savadjiev P, Alvarado JL, Westin CF, Niznikiewicz M, Nestor PG, McCarley RW, Kubicki M, Shenton ME. Localized abnormalities in the cingulum bundle in patients with schizophrenia: A Diffusion Tensor tractography study. NeuroImage Clinical. 2014; 5:93-99. [PubMed: 25003032]

Wilkinson, G. The Wide Range Achievement Test - Revision 3. Jastak Association; Wilmington, DE: 1993.

Zalesky A, Fornito A, Seal ML, Cocchi L, Westin CF, Bullmore ET, Egan GF, Pantelis C. Disrupted axonal fiber connectivity in schizophrenia. Biol Psychiatry. 2011; 69(1):80-89. [PubMed: 21035793] 

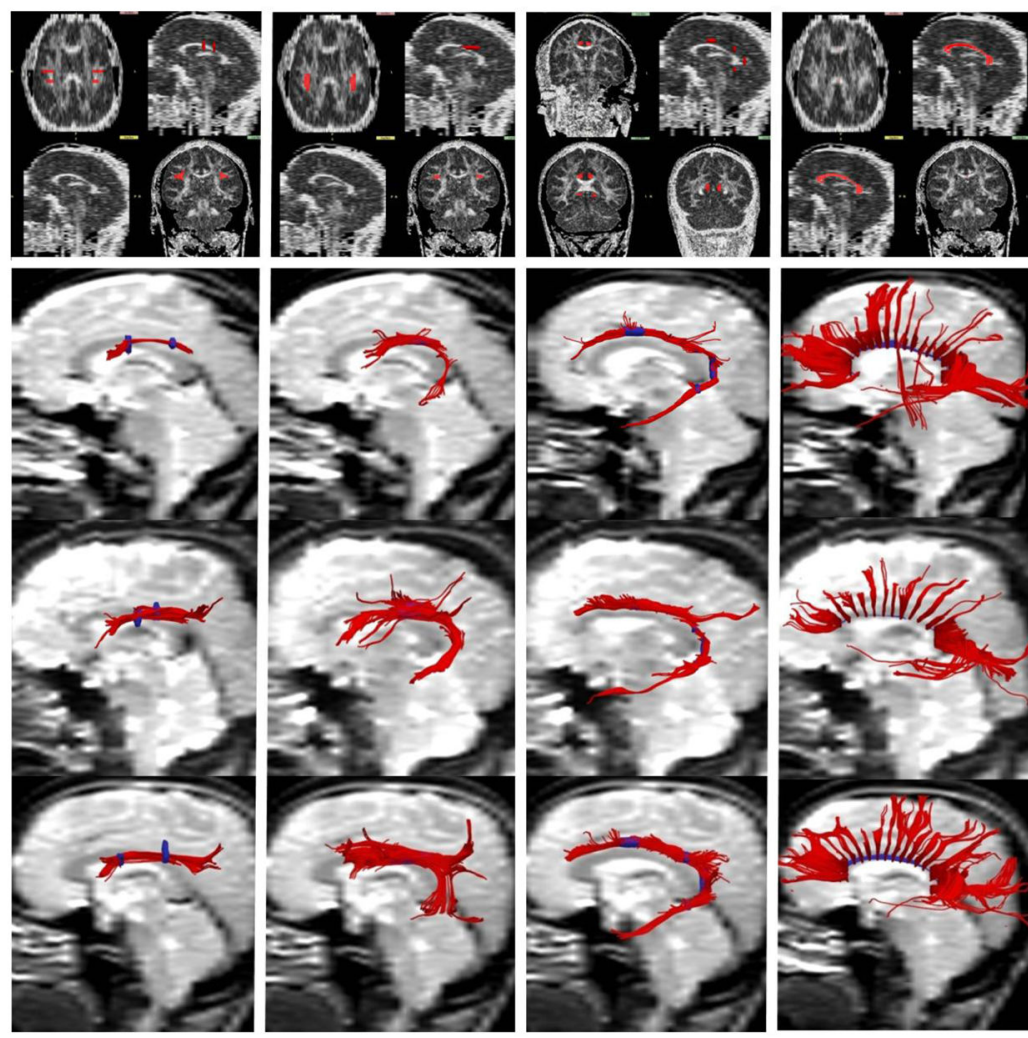

Figure 1.

Extracting the four white matter fasciculi of interest from Diffusion-Tensor Images using deterministic (streamline) tractography. The Regions-of-Interest (ROIs) and extracted fibers of the superior longitudinal fasciculus are depicted in the first column, the arcuate fasciculus in the second column, the cingulum bundle in the third column and the corpus callosum in the fourth column. The top row depicts the seeding and inclusion Regions-of-Interest (ROIs) in red, overlaid onto a single participant's FA image. The bottom three rows depict the fibers extracted from three representative participants (i.e., one from the DOC, $\mathrm{nDOC}$ and $\mathrm{HC}$ groups respectively), with the ROIs overlaid in blue. 


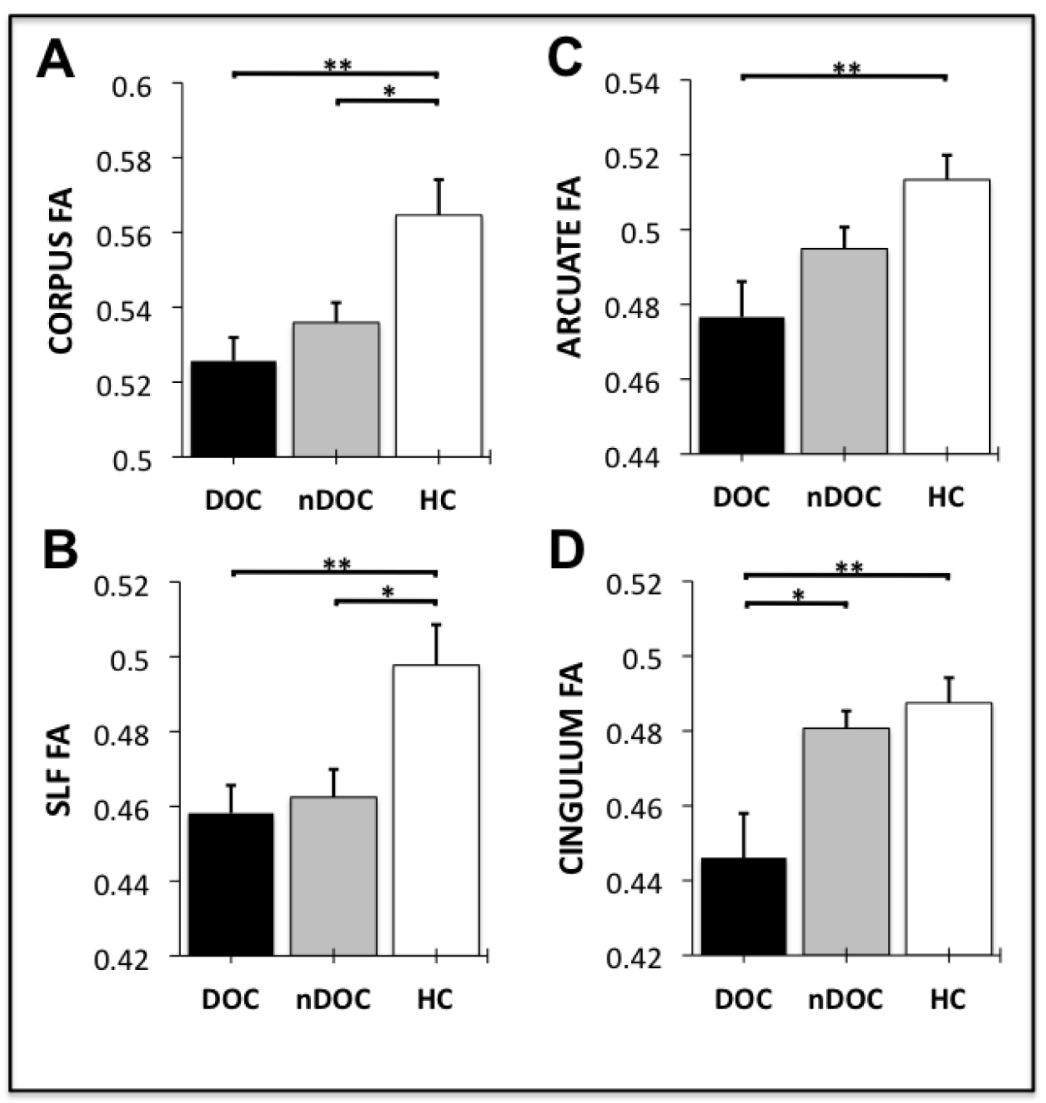

Figure 2.

Fractional Anisotropy (FA) in the corpus callosum (Panel A), superior longitudinal fasciculus (Panel B), arcuate fasciculus (Panel C) and cingulum bundle (Panel D) in schizophrenia patients with current delusions of control (DOC), schizophrenia patients without a history of delusions of control (nDOC) and healthy control participants (HC). The error bars represent the standard error of the mean. $* *=p<0.01, *=p<0.05$. 
